men. Dies dürfte zwar etwas begründungsbedürftiger sein, es ist aber auch in diesem Fall nachvollziehbar, dass man sich an einem Wochentag intensiv um sein Kind und damit dessen Erziehung kümmern möchte.

\section{Die 36 Monate müssen nicht zusammenhängend sein, aber ...} Ob der 36-Monats-Zeitraum tageweise zu zählen ist, erscheint dagegen zweifelhaft. Da 36 Monate nicht zusammenhängend genommen werden müssen, wären dies, vereinfacht berechnet, 1.080 Tage. Dies entspräche einem Tag pro Woche für die Dauer von ungefähr 20 Jahren. Eine solche Auslegung dürfte aber mit dem Wortlaut und der Intention des Gesetzes nicht vereinbar sein. Anderenfalls hätte der Gesetzgeber die Beschäftigung eines Assistenten für 144 Wochen oder 1.080 Tage erlauben können, was er aber nicht getan hat. Vertragsärzte werden daher davon ausgehen müssen, dass zwar die 36 Monate nicht zusammenhängend genommen werden müssen, dass aber eine „Stückelung" in kleinere Zeitintervalle als einen
Monat nicht zulässig ist. Wenn Praxisinhaber also einen Assistenten tageweise oder wochenweise beschäftigen, so zählt dies als Beschäftigung während eines Monats. Im Übrigen kann man aber den 36-monatigen Zeitraum frei nach den Bedürfnissen des Kindes und den eigenen Vorstellungen des Ausgleiches von Familie und Beruf bestimmen.

Bis zu welchem Alter eine Person noch als Kind im Rechtssinne gilt wird in den deutschen Gesetzen nicht einheitlich definiert. Es wird entweder von der Vollendung des 14. Lebensjahres oder des 18. Lebensjahres ausgegangen. Im Alter zwischen 14 und 18 bleibt also Raum für denkbare Auseinandersetzungen über die Beschäftigung eines Entlastungsassistenten, bis zum 14. Geburtstag des Kindes kann jeder Vertragsarzt und jede Vertragsärztin einen solchen Assistenten anstellen, wenn man sich während der „gewonnenen“ Zeit der Erziehung des Kindes widmen möchte.

Dr. Ingo Pflugmacher, Fachanwalt für Medizin- und Verwaltungsrecht, Partner der Kanzlei Busse \& Miessen, Bonn

\title{
Eigene EBM-Ziffer für das Patienten-Coaching?
}

\section{Patienten-Coaching hilft den Betroffenen und es senkt insgesamt die Kosten im Gesundheitssystem. Das gilt zumindest für Patienten, deren Behandlung besonders hohe Kosten verursacht. Das hätten Erfahrungen aus verschiede- nen Projekten gezeigt, so der Bundesverband Managed Care.}

$\mathrm{n}$ einem Positionspapier fordert der Bundesverband Managed Care (BMC) jetzt, dass das Patienten-Coaching im fünften Sozialgesetzbuch (SGB V) verankert und in den Leistungskatalog der GKV aufgenommen werden sollte. Dabei bringt der BMC vor allem wirtschaftliche Argumente vor: „Der Patient ist die größte Ressource im Gesundheitswesen, die bisher nicht genutzt ist", sagte Dr. Torsten Pilgrim aus der Projektgruppe Patienten-Coaching des BMC beim Kongress des Verbandes in Berlin. Die bestehenden selektivvertraglichen Möglichkeiten hält die Projektgruppe für nicht ausreichend. Sie fordert daher, dass das Patienten-Coaching mit einer Abrechnungsposition hinterlegt wird, die nicht nur Ärzte, sondern auch nicht-ärztliche
Leistungserbringer leisten können, die ergänzend zum Hausarzt oder betreuenden Facharzt tätig sind. Wichtig seien außerdem eine Begriffsnormierung und eine Klarstellung, wer Patienten-Coaching überhaupt anbieten dürfe.

Zur Qualitätssicherung hält der BMC eine kontinuierliche Nutzenbewertung durch den Gemeinsamen Bundesausschuss (G-BA) für nötig. Er müsse auch Ergebnisparameter für das PatientenCoaching definieren. Der Hintergrund für diesen Vorstoß ist laut BMC, dass immer mehr Menschen in Deutschland an chronischen Erkrankungen leiden, bei denen Lebensstiländerungen Folgeerkrankungen vermeiden und den Medikamentenbedarf senken könnten.

Angela Mißlbeck
Essenzielle Informationen fürTherapeuten.

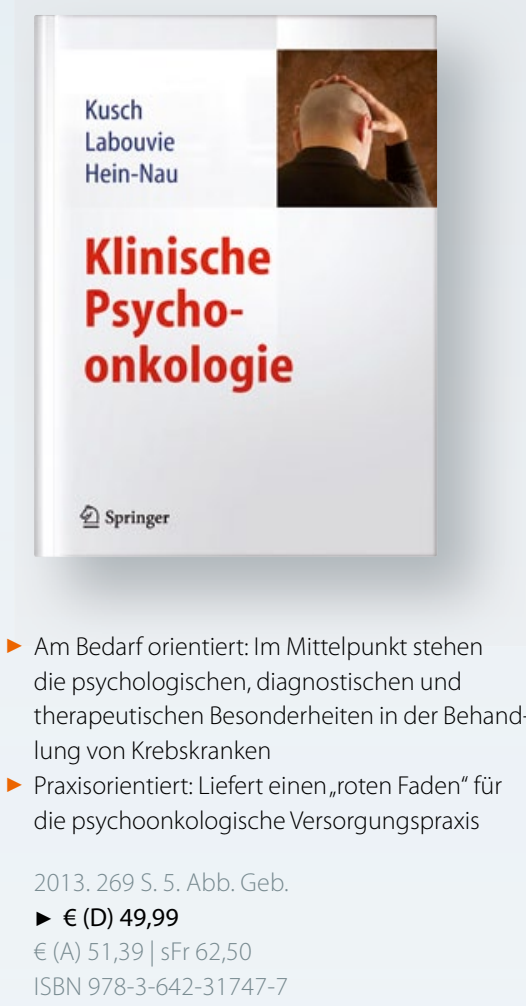

Jetzt bestellen! 